\title{
FLOMESS - Flight Load Measurement System for Sounding Rockets
}

\author{
Baader Manuel \\ Institute of Lightweight Structures \\ Bundeswehr University Munich \\ Munich, Germany \\ manuel.baader@unibw.de
}

\begin{abstract}
The future use of rockets is increasingly shifting from national to private interest. Here, primarily economic aspects decide the further development within the rocket technology. Our experiment deals with the weight reduction of the rocket structure itself in order to optimize the ratio of payload to total mass of a rocket. Here the FLOMESS project want to continue a started venture at the Bundeswehr University Munich. The FLOMESS experiment is intended to measure the structural strains during the launch of a sounding rocket. The occurring structural loads will be calculated from the measured strains. Furthermore, the system shall measure the effects of thermal strain to isolate them from the measurement. This is necessary to determine the pure structural strains. Therefore, a redundancy system of strain and temperature gauges is used on the inner surface of the experiment module. This system of relating the measured strain gauge response to the loading is taken over from previous projects on the fundament of the Skopinski method. This method was adapted for our experiment and illustrates an application of the Skopinski method to sounding rockets, where modifications being made to account all types of loadings. An accurate knowledge of the loads during the flight shall help to improve the existing semi-empirical methods for calculating these loads in different flight positions of a rocket. Since these predictions influence the design of the rocket vehicle, efficient design is achieved by reducing semi-empirical safety margins and increasing overall payload mass ratios. FLOMESS participated in the REXUS/BEXUS campaign of DLR and SNSA and, as a test of the system, the experiment was launched on the RX25 rocket on 11 March 2019. The results will be used to further develop the measurement methods.
\end{abstract}

Keywords - flight loads, flight loads measurement, structural strain, sounding rockets, Skopinski method, REXUS, RX25, failure analysis

\section{INTRODUCTION (HEADING 1)}

The idea to measure flight loads of a rocket was taken up by the Mobile Rocket Base (MORABA) part of the German Aerospace Center (DLR). After the predevelopment of a measurement module, a student team was founded in cooperation with the Institute for Lightweight Structures at the Bundeswehr University Munich (UniBw), to improve this module within the Rocket Experiments for University Students (REXUS) program. This paper deals with the development of a Flight Load Measurement System (FLOMESS) based on the theoretical foundations. In addition, it deals with the special requirements of a REXUS project and the calibration phase. Since a failure of the FLOMESS module occurred during the rocket flight, the results are presented in the form of a failure analysis.

\section{THEORETICAL FOUNDATIONS}

The Skopinski method [1] is used to calculate the resulting sectional loads at the position of the experiment module. The method assumes that a linear structural behaviour is existent. This allows, after previous calibration, the conversion of measured strains into the flight loads. This calibration procedure includes the application of defined load cases while simultaneously measuring the respective gradient of each strain output signal of the corresponding strain gauge bridge. To convert the measured strains of any number of strain gauge bridges into the required sectional loads, the calibration matrix $\beta$ must be calculated in (1). A calibration matrix can be determined from the variety of several calibration tests with different sectional loads and load cases. If as many sectional reactions are applied as there are strain gauge bridges, a determined system with a unique solution exists. The character of the Skopinski method also allows a higher number of measurement locations to be included than sectional loads, which leads to an overdetermined system. In this case, the calibration matrix can be calculated using the least squares method. This represents the general linear case of the least squares method, whereby a numerical solution of the calibration matrix can be obtained using a minimization problem. After calculating the calibration matrix, the individual strain gauge output signals are weighted by the coefficients to the corresponding load. As a result of this, combined sectional loads can be calibrated.

$$
L_{j}=\left[\beta_{i j}\right]\left\{\mu_{i}(T)-\theta_{i}(T)\right\}
$$

Decisive for this are the number of strain gauge bridges, their orientation and their application to the inner wall of the experiment module. In order to characterize different loads individually, it is necessary to be able to produce a specific sensitivity via the orientation. Therefore, the eight strain gauge rosettes in the experiment module are applied in $0^{\circ} / 90^{\circ}$ and $\pm 45^{\circ}$ arrangement and produce different output signals specific to the applied sectional load. The sectional loads during flight will then be a result from the multiplication of the calibration 
matrix and the strain output signals. In order to compensate any temperature influences, the strains will be subtracted in the post-flight phase by a determined correction function.

\section{DeVelopment of A Flight LoAd MEAsurement System}

\section{A. Hardware}

The FLOMESS experiment consists of the FLOMESS module ring with strain gauges. In order to compensate the influence of temperature, additional temperature sensors are installed. Fig. 1 shows the module ring as it was designed by Dohmjahn. It can be divided into three main hardware components:

1) Module ring with strain gauges and temperature sensors,

2) Amplifiers DMS-8 and PT-100,

3) and E-Box with MFC-2 and FLOMESS Interface Board

\section{Strain gauges and temperature sensors}

A total of four measuring points are arranged on the inside of the module ring. In order to calculate the flight loads, four measuring points with two differently arranged strain sensors each are installed. The temperature sensors are mounted in such a way that they are close enough to the strain gauges to accurately measure the surrounding temperature but still have enough distance not to influence the measurements. The RXSM of the RX-25 rocket is located directly above the FLOMESS module. This configuration leads to a small distance between the high-frequency antenna of the RXSM and the electrical wiring of the strain gauges [3]. To minimize the interference, two changes are carried out. Firstly, all unshielded wirings are shortened to reduce the antenna surface. Secondly, ferrite beads are applied to the unshielded wirings between the PCBs and amplifiers. To improve mechanical stability of the shielded wirings, four ferrite beads are gathered to a bundle and fixed to the inner wall of the module with an epoxy resin adhesive as shown in Fig. 2. Organizing all four cables of each strain gauge rosette also increases clearness of the wiring arrangement.

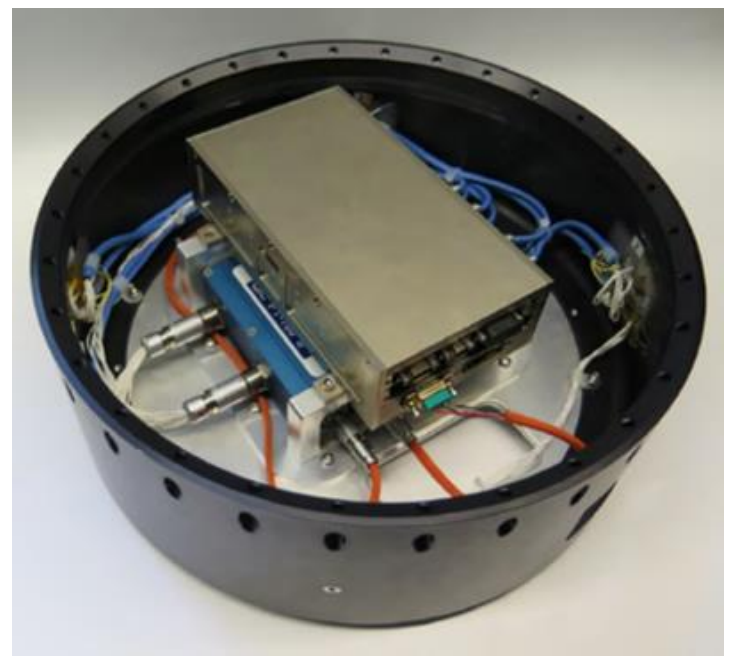

Fig. 1. FLOMESS module ring

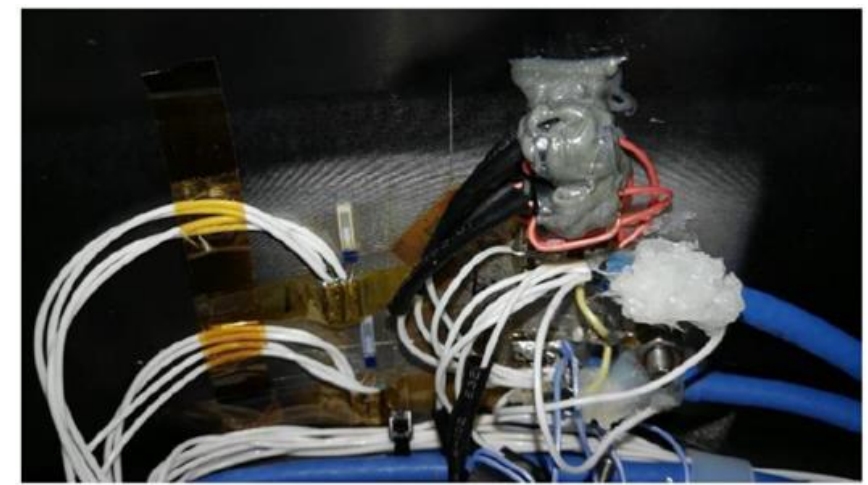

Fig. 2. Configuration of sensors with Ferrite Beads

\section{DMS-8 and PT-100 amplifiers}

The DMS- 8 module is used as the amplifier module for the strains. The PT-100 module is used to measure the temperature. Both are measurement modules originally used in automobile production. The analog signal is amplified and converted into a digital signal in an A/D converter.

\section{E-Box with MFC-2 and FLOMESS Interface Board}

For mounting in the FLOMESS ring and for protection against physical influences and radio waves the MFC-2 and the FLOMESS Interface Board are located in an E-Box. The main task of the MFC-2 is the signal processing. The MFC-2 supports various interfaces, of which the CAN bus and RS-422 connector were used due to compatibility with the amplifiers and the REXUS Service Module (RXSM). The MFC-2 is connected to RXSM via the FLOMESS Interface Board. The $28 \mathrm{~V}$ power voltage of the RXSM is fed to a TRACO 4 Power step-down converter with an output voltage of $5 \mathrm{~V}$ with a maximum current of $2000 \mathrm{~mA}$. A common mode filter is connected to the power supply generating a ripple free power voltage. Optocouplers are used for the galvanic isolation of the rocket signals. Voltage Peaks over $28 \mathrm{~V}$ have no influence on the connected hardware. Returning voltage peaks from the FLOMESS electrical components are blocked because of the galvanic isolation.

\section{B. Software}

The software is divided into two sections, the onboard software on the FLOMESS module ring and the Ground Control Station (GCS).

\section{Onboard Software}

The onboard software is part of the measurement system in the data processing function. In addition, the software is processing the rocket signals including the timeline. At least, it is responsible for the up- and downlink. To program the MFC2 the programming language $\mathrm{C}++$ is used together with the RODOS real-time operating system, including modifications and tools kindly provided by MORABA. The core of the software was already finished by MORABA as part of the master thesis of Karl Domjahn [2]. 


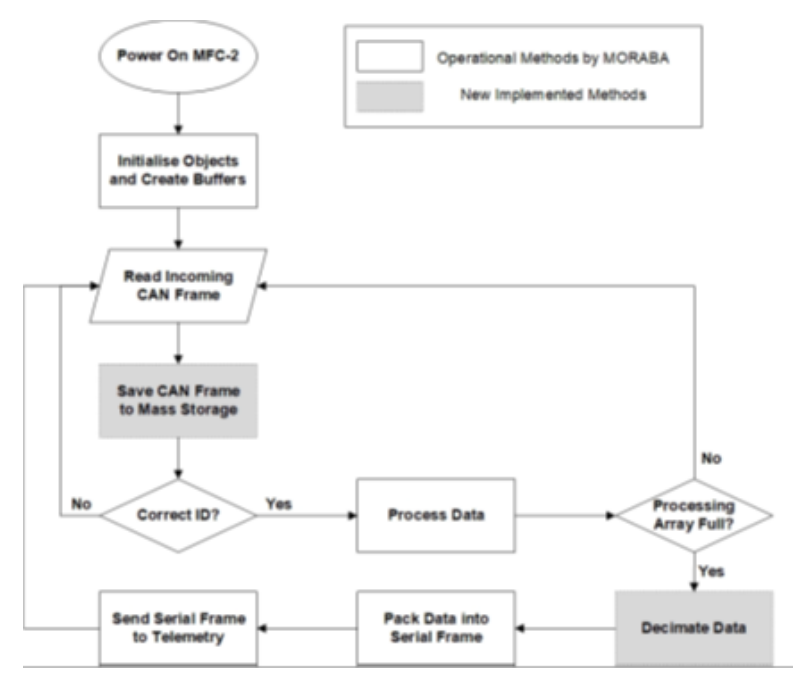

Fig. 3. Software Architecture Flowchart

Data Processing: The MFC-2 is connected to the DMS- 8 and PT-100 amplifiers through an internal CAN bus for reading out and setting. The CAN message consists of three parts: a timestamp, an ID and the measurement data. To sort the data into the two classes DMS and PT100, the IDs of the CAN messages are used. This function has already been completed by MORABA and Domjahn [2]. As shown in Fig. 3, the functions "Decimate Data" and "Save CAN Frame to Mass Storage" are newly implemented. The data sent to the GCS is decimated using a buffer. The decimation rate is used as the length of a buffer. When this buffer is full, the first measurement is sent. The number of data packets to be decimated depends on the sample rate of the DMS- 8 amplifiers and must be set before the rocket launch. The second function is "Save CAN Frame to Mass Storage" and the following solution was developed. Onboard the data is stored on an SD Card. The message size of a stored data packet is 18 bytes per CAN message. At a sampling rate of $1 \mathrm{kHz}$ for the DMS-8 amplifier and $10 \mathrm{~Hz}$ for the PT-100 amplifier it results in a total memory requirement of about $80^{\prime} 000 \mathrm{kB}$ by a recording time of 2250s. Further system data of the amplifiers and the MFC-2 are stored, but their memory requirements are less than $10 \%$ of the total data. The measured data can be read out after the flight by executing a function via the programming interface.

Rocket Signals: Since direct control by the uplink is only possible before LO, the software must run automatically. The RXSM provides the following three rocket signals for this purpose: Start of Data Storage (SODS), Start of Experiment (SOE) and Lift Off (LO). In the FLOMESS experiment the signals are implemented as follows. As soon as a signal is triggered, the voltage difference is noticed by the MFC- 2 . With a program loop it is checked which signal is activated. The signal whose circuit is no longer switched off is seen as activated. Then the following functions are executed in the software program in accordance with the timeline.

Downlink: The data is sent from the FLOMESS module to the GCS via the RXSM, TM, SCIENCE-NET. The requirements of the RX User Manual include the max baud rate of $30 \mathrm{kbit} / \mathrm{s}$ [3]. For the FLOMESS experiment the downlink packets are sent as 20 Byte datagrams (Fig. 4). The start and end of the data packets are predefined in the RX User Manual. In order not to exceed the baud rate, a data decimation takes place on the MFC-2, as already mentioned. The sampling rate of $1 \mathrm{kHz}$ is reduced to $83 \mathrm{~Hz}$ and this leads to a download rate of about $27 \mathrm{kbit} / \mathrm{s}$.

\begin{tabular}{|c|c|c|c|c|c|c|c|c|c|c|c|c|c|c|}
\hline 1 & 2 & 3 & 4 & 5 & \begin{tabular}{l|l}
6 & 7
\end{tabular} & 8 & \begin{tabular}{l|l}
9 & 10
\end{tabular} & \begin{tabular}{|l|l|l|}
11 & 12
\end{tabular} & \begin{tabular}{l|l}
13 & 14
\end{tabular} & \begin{tabular}{l|l}
15 & 16
\end{tabular} & 17 & 18 & 19 & 20 \\
\hline$\frac{\bar{u}}{\sum_{i}}$ & 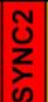 & $\begin{array}{l}\text { 을 } \\
\text { 五 }\end{array}$ & 方 & & $\underset{\sum}{\underline{E}}$ & & $\underset{\mathbb{S}}{\stackrel{5}{4}}$ & 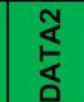 & $\underset{\mathbb{L}}{\stackrel{m}{\leftarrow}}$ & 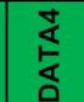 & $\frac{0}{\alpha}$ & 这 & U & 晟 \\
\hline
\end{tabular}

Fig. 4. Downlink Protocol

Uplink: The uplink transmission path differs slightly from the downlink. The commands are sent from the own GCS to the FLOMESS module via the SCIENCE-NET, the EGSE and the RXSM. The data format is equal to the downlink except the missing timestamp. The commands concerning either the amplifiers, the memory card or the MFC-2 each receive different IDs. In the data area, values can be transferred to the MFC-2

\section{Ground Control Station}

The GCS is divided into three parts: A real-time display of the downlink data, a control console and a storage of the incoming data. The ground control software will be implemented as a client-server system: A server program receives the packet data from the ground control infrastructure. It is written in the Crystal language, an improved variant of Ruby. This server program stores every received packet in a database and forwards it to a frontend running on a webserver. This frontend displays the raw data and calculated flight loads.

Realtime Display: The realtime display is used to monitor the experiment. Status values are displayed for all of them in the lower right corner. This includes the server time and status of the server, telemetry and rocket signals. The first display shows the raw data of all channels (Fig. 5). The digital 16-bit numbers of all measuring locations are plotted over time in four graphs. The upper two graphs show the measured strains. The lower two graphs show the temperatures.

Control Console: The control console is implemented like the live display via the webfrontend (Fig. 5). Besides the commands to control the amplifiers, the SD card methods can also be executed. In addition, there is the option to read out the SD card and download the data via the MFC-2 for post-flight analysis. A dropdown menu is useful for commands that pass certain values, as this reduces the number of buttons and keeps the control console clear.

Data Storage: The data storage on the GCS is used as a backup for the onboard memory card. All incoming data is stored on a web-based database. The data can be received from a server connected PC via a web browser. The page is then saved as a text file. Since the ID of the amplifier channels and the measurement data are available in the downlink data format as in the CAN data, the data can be quickly read out during the post-flight analysis and sorted according to the measurement locations. 


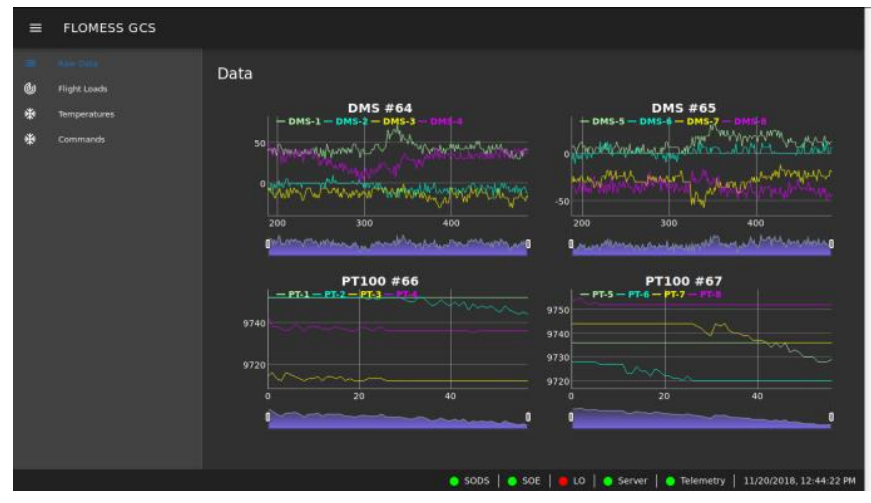

Fig. 5. Ground Control Station: Rawdata

\section{CAlibration Phase}

\section{A. Testing}

For the calibration phase, the test facilities at the Institute for Lightweight Structures are used. The aim is to calibrate each section load independently of each other. Each test take place under static load.

Axial Force: To calibrate the normal force $\mathrm{Nx}$, various pressure tests are performed using a hydraulic cylinder. By means of centric or eccentric pressure different load cases have been simulated. In calibration tests with eccentric pressure, several lever arms are selected from the force introduction point to cover different bending moments. Thirty tests are carried out to describe this type of load.

Shear Forces and Bending Moments: The calibration of transverse forces $\left(\mathrm{Q}_{\mathrm{y}}\right.$ and $\left.\mathrm{Q}_{\mathrm{z}}\right)$ include the simultaneous calibration of bending moments $\left(\mathrm{M}_{\mathrm{y}}\right.$ and $\left.\mathrm{M}_{\mathrm{z}}\right)$. Therefore, the rocket structure is attached to a test bench using an adapter plate. A shear force is then applied to the structure via a hydraulic cylinder. The resulting moment can be modified by variation of the position of the experiment module in axial direction. A total of nine tests are carried out for this experiment setup, with three height variations taking place with subsequent rotation of $0^{\circ}, 45^{\circ}$ and $90^{\circ}$.

Bending-Torsion: The calibration of a pure torsional moment is difficult with respect of the available test equipment. To allow calibration of the torsional moment $\mathrm{Mt}$, the cutouts of a dummy module are used. In contrast to the structure for calibrating shear force and bending moment, the force application have to be done in order to generate a torsional moment around the longitudinal axis. Therefore, an eccentric load is introduced by a wooden beam, which is inserted through the cutouts and loaded on one side with a transverse force. Nine tests are performed under the same variation as the shear force and bending moment calibration.

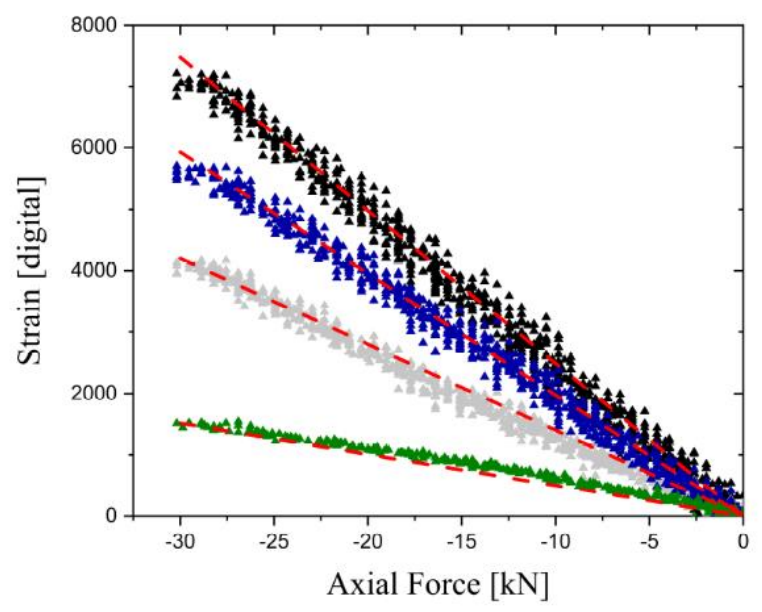

Fig. 6. Evaluation of the Strain Gradients

\section{B. Analysis Process}

After performing all the calibration tests, the measured data must be analysed in focus of the produced strain gradients. The strain gradients are required for the calculation of the matrix. The gradients are determined by linear approximation according to the assumption of linear structural behaviour. Load application and reduction result in two gradients that ideally overlap. For the evaluation of the calibration tests only the load introduction, i.e. the rising edge of the gradient, is considered. Fig. 6 confirms the correct assumption of linear structural behaviour. The transposed calibration matrix $\beta^{\mathrm{T}}$ can be calculated from the strain gradient of each strain measuring location and the corresponding load vector using (2). The vectors of the strain gradients $\mu$ and the loads $L$ are converted into matrices by assembling the vectors column by column from the right.

$$
[\beta]^{T}=\left[[\mu][\mu]^{T}\right]^{-1}\left[[\mu][L]^{T}\right]
$$

In order to determine a direct deviation, the forces in the load vector of the respective calibration tests are normalized to one to calculate the calibration matrix. The resulting moments then are calculated from the normalized force multiplied by the corresponding lever arm. To be able to carry out a comparison of the individual section loads, the loads are summed over all calibration tests in which they are calibrated and then divided by this number. This is also done with the calibration loads.

\section{Temperature Influence}

In addition to the calibration of the system in order to be able to determine structural loads from strains, a distinction has to be made between thermal and mechanical strain in the event of an expected temperature increase of the rocket structure during flight. The disturbance of the thermal expansion is influenced by the factors of the different application and orientation of the strain gauge rosettes. By using a PT-100 temperature sensor close to each individual strain gauge bridge, correction functions for transient heating can be determined. The experiment involves heating the structure to the shut-off temperature and then cooling it down to the initial temperature. 
Tire heating blankets are used to heat the rocket structure. At the same time, the strains and the associated temperatures are measured to describe the effect of the thermal strain. The result shows that the strain gauge rosettes are influenced by temperature changes depending on their orientation. In order to consider the temperature strain, correction functions are derived from the determined strain-temperature graphs for each individual strain measurement rosette. The correction functions are a third degree polynomial. They are difficult to validate for the real flight, because they can only be confirmed on the basis of the performed tests. It is also unclear whether the functions cover a larger interval than can be tested. The determined correction functions can lead to a partial isolation of the temperature influence under combined mechanical and thermal load.

\section{FAILURE ANALYSIS}

After a system failure of the FLOMESS module occurred during the rocket launch of the RX-25 rocket, no calculated flight loads according to the Skopinski method can be presented here as a result. Instead, a failure analysis is carried out here with the purpose of localizing the main cause and preventing such a system failure during future flights of the FLOMESS module. A failure analysis consists of the following steps [4]:

1) Information gathering,

2) Failure definition,

3) Creating a fault tree with a Fault Tree Analysis (FTA),

4) Investigation of the events with a Failure Mode Assessment and Assignment Matrix (FMA\&AM)

5) Converging to the main cause.

The different steps are performed using the FLOMESS experiment as an example.

\section{A. Information Gathering}

The first step is also one of the most important. The more information about the failure that has occurred is collected, the more accurate the subsequent investigation of the failure can be. Recommended by [4], at least the following information should be collected:

- All TM recorded by ground station, including the raw and processed data.

- All recorded commands sent to the experiment

- All telemetry available from the RXSM

- All the assembly documentation

In addition, photos are used, which were taken immediately after the recovery of the RX-25 rocket and show the FLOMESS module with all connectors from different sides.

\section{B. Failure Definition}

The failure definition should describe as exactly as possible the failure as it occurred. It should be avoided to come to rash conclusions about possible causes in this step of the error analysis. The following failure occurred during the FLOMESS experiment: "From a moment of approx. 1 second before $L O$ no measurement data arrived at the GCS. Furthermore, no data was received during the entire rocket flight."

\section{Fault Tree Analysis}

FTA is a top-down symbolic logic model generated in the failure domain. This model traces the failure pathways from a predetermined, undesirable condition or event, called the TOP event, of a system to the failures or faults (fault tree initiators) that could act as causal agents."[5] A fault tree consists of various events and gates that the events pass through to trigger the next event. Although many event and gate symbols exist, most fault trees can be constructed with the following four symbols: TOP or Intermediate event, inclusive OR gate, AND gate, and basic event. The symbols and descriptions of these events and gates are shown in TABLE I. Fig. 7 shows the finished FLOMESS fault tree. The complex fault tree is easier to understand because the individual events are coloured according to the categories general, hardware, software and environmental influences. Since only inclusive or gates are used, it is not required to specify the gates.

TABLE I. FTA SYMBOLS

\begin{tabular}{|l|l|l|}
\hline \multicolumn{1}{|c|}{ Nymbol } & \multicolumn{1}{|c|}{ Description } \\
\hline $\begin{array}{l}\text { Event (TOP or } \\
\text { intermediate) }\end{array}$ & $\begin{array}{l}\text { This is the event to which failure } \\
\text { paths of lower level events lead. }\end{array}$ \\
\hline & $\begin{array}{l}\text { Inclusive OR } \\
\text { gate }\end{array}$ & $\begin{array}{l}\text { An output occurs if one or more } \\
\text { inputs exist. Any single input is } \\
\text { necessary and sufficient }\end{array}$ \\
\hline
\end{tabular}

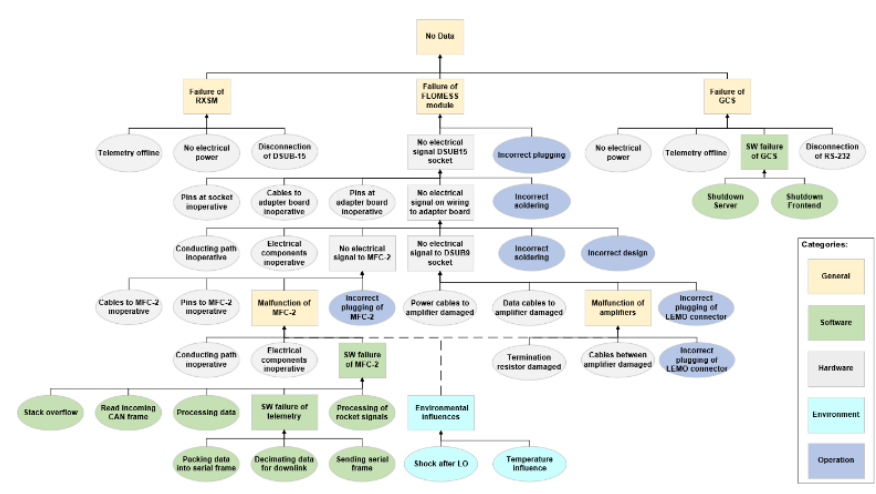

Fig. 7. FTA for FLOMESS 


\section{Failure Mode Assessment and Assignment Matrix}

All basic events, also known as leaves, are now placed in the FMA\&AM. TABLE II. shows the possible FMA\&AM for the different categories from the FTA. Assessment is simply whether the event is still a credible failure point or has been discounted by an investigation. Investigation actions should be sorted by their degree of reversibility.

TABLE II. FMA\&AM FOR FLOMESS CATEGORIES

\begin{tabular}{|l|l|l|}
\hline \multicolumn{1}{|c|}{ Event } & Assessment & \multicolumn{1}{c|}{ Investigation Action } \\
\hline Operation & & $\begin{array}{l}\text { 1. Visual check } \\
\text { 2. Check of documentation/data } \\
\text { 3. Functionality test }\end{array}$ \\
\hline Hardware & & $\begin{array}{l}\text { 1. Visual check } \\
\text { 2. Check of datasheets }\end{array}$ \\
& & $\begin{array}{l}\text { 3. Functionality test } \\
\text { 4. Electrical function check }\end{array}$ \\
\hline Software & & $\begin{array}{l}\text { 1. Confirm checklist } \\
\text { 2. Functionality test }\end{array}$ \\
& & $\begin{array}{l}\text { 3. Check of SW code } \\
\text { 4. Modify parts of SW code }\end{array}$ \\
\hline & & 2. Test with different Temperatures \\
& &
\end{tabular}

Investigations carried out showed the following results:

1) By analysing the existing flight data and the own flight procedures it can be excluded that the failure is located at the GCS or the RXSM.

2) By the visual check and with the available postflight photos already some basic events can be excluded, e.g. incorrect plugging.

3) By functionality tests, the failure caused by hardware components can be excluded.

4) By several function tests at different temperatures, the influence of temperature can be excluded.

Therefore, the failure is in the software.

\section{E. Converging to the main cause}

In the last step, the main cause is investigated in more detail so that it can be removed in the future. If the time stamps of the last messages sent are considered, it results that at 3.5 seconds before LO the last measurement data were sent. However, the last message sent was the LO signal processed on the MFC-2. A very similar situation could be simulated in the laboratory by running the MFC-2 until apparently no more measurement data was sent. The RXSM simulator then triggered the LO signal with a delay of a few seconds. The following conclusions can be reached:

1) Not all the software fails,

2) The failure is probably related to a stack overflow,

3) The communication between MFC-2 and amplifiers seems to be interrupted.

In order to further locate the failure, the various storage operations should be investigated more closely in the future. The priority is to look at the functions for reading out the can data.

\section{ACKNOWLEDGMENT}

The entire team and the author of this article would like to thank those responsible for the program and the sponsors. The main actors are ESA, DLR, ZARM, SSC, SNSA and our initiators for the project MORABA. This gave us the opportunity to apply our studies practically and to participate in such a space program like REXUS. Also, we would like to express our special thanks to our sponsors from industry such as ZT Management Holding $\mathrm{GmbH}$ and CAETEC GmbH. Finally, we would like to thank our university and the trust of our supervising professor for them continued support.

\section{REFERENCES}

[1] T. H. Skopinski, W. S. Jr Aiken, and W. B. Huston, "Calibration of Strain-Gage installations in aircraft structures for the measurement of flight loads," NACA Report 1178, 1954

[2] K. Domjahn, "Design of a Flight Load Measurement System for sounding rockets," Bachelor-/Masterthesis, University of Queensland: Faculty of Engineering, Architecture and Information Technology, 2016, unpublished

[3] K. Schüttauf, "REXUS User Manual," Technical report, EuroLaunch, 2017

[4] A. Kinnaird, "Failure analysis for REXUS/BEXUS experiments," ESRANGE, Sweden: SSC, 2018, unpublished

[5] K. Everhart, R. Stevens, N. Babbit III, P. Clemens, L. Stout, \& B. Goldberg, "System engineering "Toolbox" for design-oriented engineers," Marshall Space Flight Center, Alabama: NASA, 1994 